\title{
Uterine artery embolization for uterine arterio-venous malformation
}

\section{Haritha Sagili ${ }^{1}$, Nagarajan Krishnan ${ }^{2}$, Anish Keepanasseril ${ }^{1}$, Sathiya Priya ${ }^{1 *}$, Sunilkumar Devaraj ${ }^{2}$}

\begin{abstract}
${ }^{1}$ Department of Obstetrics and Gynecology, Jawaharlal Institute of Postgraduate Medical Education and Research, Puducherry, India

${ }^{2}$ Department of Radio-diagnosis, Jawaharlal Institute of Postgraduate Medical Education and Research, Puducherry, India
\end{abstract}

Received: 22 May 2021

Accepted: 18 June 2021

*Correspondence:

Dr. Sathiya Priya,

E-mail: sathiyapriyasubburaj@gmail.com

Copyright: ( ) the author(s), publisher and licensee Medip Academy. This is an open-access article distributed under the terms of the Creative Commons Attribution Non-Commercial License, which permits unrestricted non-commercial use, distribution, and reproduction in any medium, provided the original work is properly cited.

\begin{abstract}
Uterine arteriovenous malformations (AVM) as a cause of abnormal uterine bleeding are listed under "not otherwise classified" in Palm-Coein classification, is an abnormal communication between an artery and vein without an intervening capillary bed resulting in increased pressure and high velocity in the venous system. We report the case of heavy menstrual bleeding in a thirty-five-year-old primiparous lady for five years with fourteen weeks sized uterus and six gm hemoglobin. Ultrasound revealed an enlarged uterus with tubular spaces and color doppler showed tubular anechoic structures, both within the myometrium, with low resistance and high-velocity pattern suggestive of uterine arteriovenous malformations. Because of nonresponse to medical management, uterine artery embolization was carried out following which she had significant reduction in menstrual bleeding emphasizing it as a differential diagnosis in all cases presenting with heavy menses. Uterine artery embolization appears to be an effective modality of treatment especially in women whose wish to preserve fertility.
\end{abstract}

Keywords: Uterine artery embolization, Heavy menstrual bleeding, Uterine arterio-venous malformation

\section{INTRODUCTION}

Heavy menstrual bleeding can be due to a multitude of causes; the etiology being listed in the acronym Palm Coein. ${ }^{1}$ We present an unusual cause of heavy menstrual bleeding in a 35-year-old woman due to uterine arteriovenous malformations. Uterine arteriovenous malformations-a rare but life-threatening condition, is an abnormal direct communication between an artery and vein without an intervening capillary bed resulting in increased pressure and high velocity in the venous system. Nowadays more cases are reported due to increased availability of imaging facilities. Treatment varies from simple medical management to embolization and hysterectomy.

\section{CASE REPORT}

A 35-year-old para 1 live 1, last childbirth 13 years back presented with heavy menstrual bleeding with 30-day regular cycles with prolonged bleeding for 6 days soaking 4 to 5 pads per day with intermenstrual spotting or bleeding for the last 5 years. There was no history of uterine instrumentation or surgery. On examination she had severe pallor, bimanual pelvic examination revealed an anteverted, enlarged uterus approximately 14 weeks size, mobile and fornices were free. She had a hemoglobin of $6 \mathrm{gm} / \mathrm{dl}$. Ultrasound of uterus showed enlarged uterus with tubular spaces within the myometrium (Figure 1) and color doppler showed serpiginous structures within the myometrium with low resistance, high-velocity pattern 
(Figure 2) suggestive of uterine AV malformation. CT angiography revealed bilateral uterine arteries with hypertrophied branches and with abnormal vascular in the uterus and delayed draining veins (left $>$ right). She received blood transfusion and was started on Iron tablets. Heavy menstrual bleeding was unresponsive to medical treatment (oral contraceptives for 3 months); hence digital subtraction angiography and uterine artery embolization were planned. Under fluoroscopic guidance left uterine artery embolization was carried out using gel foam and right uterine embolization was deferred due to vessel spasm and no complications in short term. On follow up after a month, she had a significant reduction in menstrual bleeding. Uterine AV malformation is an unusual yet curative cause of AUB which comes under not otherwise classified of palm Coein.

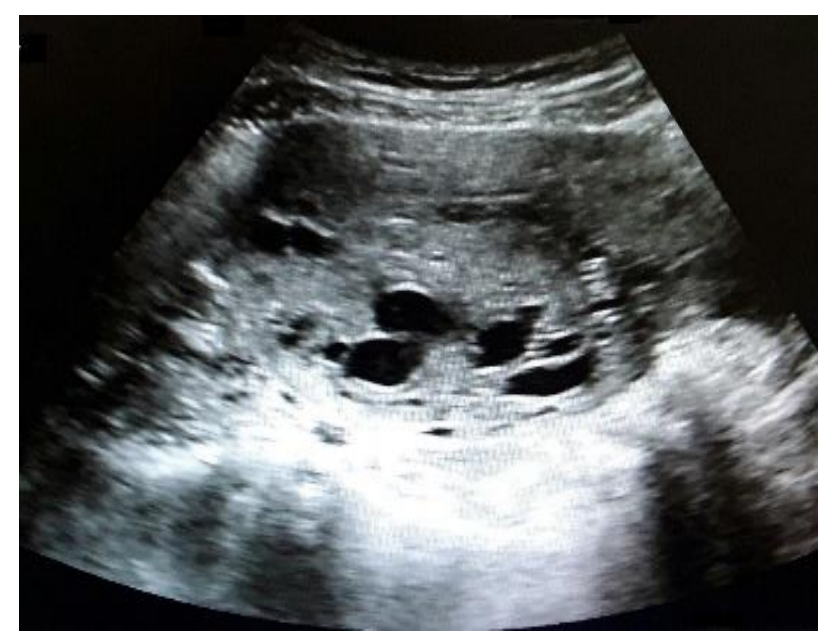

Figure 1: Greyscale ultrasound of a 35-year-old primiparous woman of uterus with multiple spongy anechoic spaces in the posterior myometrium with normal endometrial thickness.

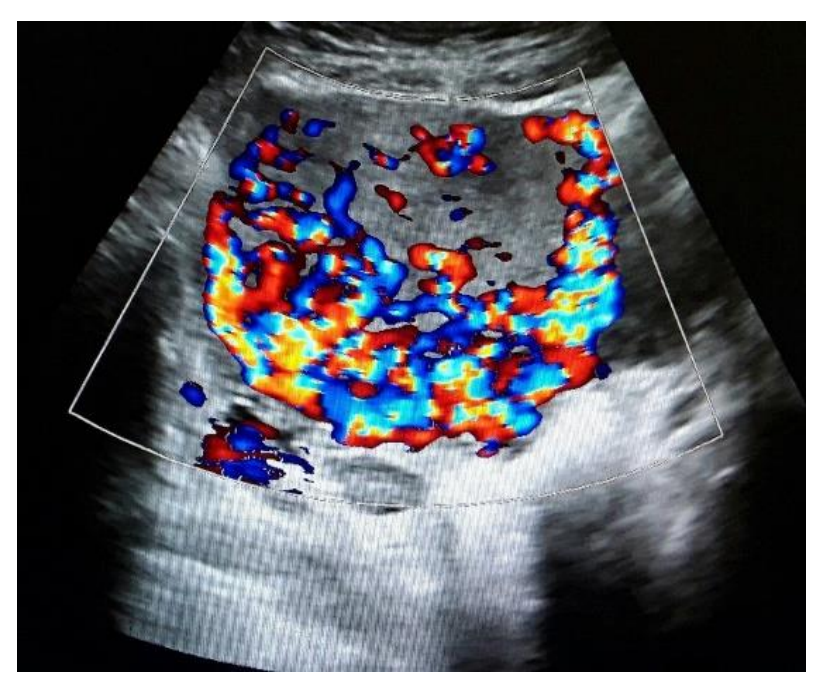

Figure 2: Color Doppler sonography of a vascular mass in posterior myometrium with turbulent multidirectional flow showing color mosaic pattern.

\section{DISCUSSION}

Fewer than 100 cases have been reported in the literature with a rough incidence of $4.5 \%$ by O'Brien et al. ${ }^{2}$ Uterine vascular malformations are hyper-vascular lesions with turbulent flow identified by early filling on angiography or pathological examination of the uterus after hysterectomy. ${ }^{3}$ Uterine AVM can be congenital or acquired $^{4,5}$ While congenital AVM is due to embryological failure in differentiation and has a central nidus containing both arteries and veins, typically extends beyond uterus into the surrounding pelvis and present at multiple sites. Acquired AVM is characterized by a single arteriovenous communication between the branches of the uterine artery and myometrial venous plexus. Acquired lesions are more common in reproductive-age women in the postpartum period and post-abortal period either following a spontaneous miscarriage or curettage. Also, associated with infection, inflammation, retained products of conception, gestational trophoblastic disease, pelvic trauma, and exposure to diethylstilbestrol. ${ }^{6}$

Acquired AVM commonly presents as unexplained, intermittent, and heavy bleeding per vaginum after delivery or surgical procedures on the uterus while congenital presents as heavy menstrual bleeding. The other symptoms include pelvic pressure or discomfort, urinary symptoms, dyspareunia, seldomly vulvar varices and lower limb venous congestion, anemia, hypotension, and rarely high output cardiac failure.

Diagnosis is done by grayscale ultrasound which demonstrates multiple tubular anechoic or hyperechoic areas within the myometrium. ${ }^{2,4}$ Color doppler shows a vascular mass with multidirectional flow, color mosaic patterns, high velocity, low resistance flow, low pulsatility of the arterial waveform, and pulsatile high-velocity venous waveform. ${ }^{5}$ Digital subtraction angiography (DSA) is the gold standard for diagnosis but used only before embolization since it is invasive and the findings are bilateral hypertrophy of the uterine arteries feeding a tortuous mass, with early drainage into massive hypertrophic veins. ${ }^{5}$ MRI shows multiple serpiginous flow-related signal voids within the lesion and is indicated for those lesions extending beyond the uterus. Dynamic contrast-enhanced MRA and CT angiogram can also be used for diagnosis and preprocedural planning.

Management depends on the patient's age, desire to preserve fertility and hemodynamic stability. Nowadays, angiographic uterine embolization is the preferred treatment because it is minimally invasive and preserves fertility in women with potentially life-threatening bleeding who are hemodynamically unstable. ${ }^{7}$ One or both uterine arteries can be embolized using polyvinyl alcohol, gel foam, or glue. Complications include pain, postembolization syndrome, perineal skin sloughing, fistulas, and neurological deficits. The success rate reported by various case series range from 79-92\%. 8.9 Reports documented in the literature with pregnancies 
following UAE though there are risks of placental accreta, fetal growth restriction, preterm labor, uterine rupture, postpartum hemorrhage, and remote chances of ovarian failure. ${ }^{7,10}$ Hysterectomy remains the definitive treatment especially in women with uncontrolled bleeding who do not wish to preserve fertility, in resource-poor settings where facilities are not available, or in whom embolization fails and in postmenopausal women. ${ }^{9}$ Those with a single bleeding episode or hemodynamically stable who are willing to follow up; expectant or medical management can be tried. Spontaneous resolution has also been reported in few cases. ${ }^{11}$ The medical management includes oral contraceptives, estrogen and progestins, danazol, gonadotropin-releasing hormone agonists. Other surgical management reported less frequently are surgical removal of AVM by local resection of the myometrium, coagulation of AVM under hysteroscopy guidance, laparoscopic bipolar coagulation of uterine vessels, and ligation of uterine and internal iliac vessels. Dilatation and curettage are contraindicated for those suspected with uterine AVM.

The cause of bleeding in our patient might be due to congenital AVM. Since she was symptomatic with anemia after a trial of medical therapy, embolization was done with immediate relief in symptoms, shorter hospital stays, fewer complications, and has a prospect for fertility.

\section{CONCLUSION}

Uterine AV malformation should be thought of as a differential diagnosis in all cases presenting with heavy menstrual bleeding. A multidisciplinary approach including the availability of conservative alternatives to hysterectomy is needed to provide optimal care. Uterine artery embolization appears to be an effective modality especially in women who wish to preserve fertility.

\section{Funding: No funding sources}

Conflict of interest: None declared

Ethical approval: Not required

\section{REFERENCES}

1. Munro MG, Critchley HOD, Fraser IS. The two FIGO systems for normal and abnormal uterine bleeding symptoms and classification of causes of abnormal uterine bleeding in the reproductive years: 2018 revisions. Int J Gynecol Obstetr. 2018;143(3):393408.

2. O'Brien P, Neyastani A, Buckley AR, Chang SD, Legiehn GM. Uterine arteriovenous malformations: from diagnosis to treatment. J Ultrasound Med. 2006;25(11):1387-92.

3. Grivell RM, Reid KM, Mellor A. Uterine arteriovenous malformations: a review of the current literature. Obstet Gynecol Surv. 2005;60(11):761-7.

4. Polat P, Suma S, Kantarcý M, Alper F, Levent A. Color Doppler US in the evaluation of uterine vascular abnormalities. Radiographics. 2002;22(1):47-53.

5. Fleming H, Ostör AG, Pickel H, Fortune DW. Arteriovenous malformations of the uterus. Obstet Gynecol. 1989;73(2):209-14.

6. Hickey M, Fraser IS. Clinical implications of disturbances of uterine vascular morphology and function. Baillieres Best Pract Res Clin Obstet Gynaecol. 2000;14(6):937-51.

7. Chen L-K, Yang B-L, Chen K-C, Tsai Y-L. Successful Transarterial Embolization of Uterine Arteriovenous Malformation: Report of Three Cases. Iran J Radiol. 2016;13(1):e15358.

8. Kwon JH, Kim GS. Obstetric iatrogenic arterial injuries of the uterus: diagnosis with US and treatment with transcatheter arterial embolization. Radiographics. 2002;22(1):35-46.

9. Yang JJ, Xiang Y, Wan XR, Yang XY. Diagnosis and management of uterine arteriovenous fistulas with massive vaginal bleeding. Int $\mathbf{J}$ Gynaecol Obstet. 2005;89(2):114-9.

10. Eling R, Kent A, Robertson M. Pregnancy after uterine arteriovenous malformation-case series and literature review. Australas J Ultrasound Med. 2012;15(3):87-96.

11. Peitsidis P, Manolakos E, Tsekoura V, Kreienberg R, Schwentner L. Uterine arteriovenous malformations induced after diagnostic curettage: a systematic review. Arch Gynecol Obstet. 2011;284(5):1137-51.

Cite this article as: Sagili $\mathrm{H}$, Krishnan N, Keepanasseril A, Priya S, Devaraj S. Uterine artery embolization for uterine arterio-venous malformation. Int J Reprod Contracept Obstet Gynecol 2021;10:2902-4. 